\title{
Recognizing the breathing resistances of wearing respirators from respiratory and sEMG signals with artificial neural networks
}

DOI:

10.1016/j.ergon.2017.02.001

\section{Document Version}

Accepted author manuscript

Link to publication record in Manchester Research Explorer

Citation for published version (APA):

Yang, Z., Chen, Y., Wang, J., \& Gong, R. (2017). Recognizing the breathing resistances of wearing respirators from respiratory and SEMG signals with artificial neural networks. International Journal of Industrial Ergonomics, 58, 47-54. https://doi.org/10.1016/j.ergon.2017.02.001

Published in:

International Journal of Industrial Ergonomics

\section{Citing this paper}

Please note that where the full-text provided on Manchester Research Explorer is the Author Accepted Manuscript or Proof version this may differ from the final Published version. If citing, it is advised that you check and use the publisher's definitive version.

\section{General rights}

Copyright and moral rights for the publications made accessible in the Research Explorer are retained by the authors and/or other copyright owners and it is a condition of accessing publications that users recognise and abide by the legal requirements associated with these rights.

\section{Takedown policy}

If you believe that this document breaches copyright please refer to the University of Manchester's Takedown Procedures [http://man.ac.uk/04Y6Bo] or contact uml.scholarlycommunications@manchester.ac.uk providing relevant details, so we can investigate your claim.

\section{OPEN ACCESS}




\title{
Recognizing the breathing resistances of wearing respirators from respiratory and sEMG signals with artificial neural networks ${ }^{\text {th }}$
}

\author{
Yumiao Chen $^{\mathrm{a}}$, Zhongliang Yang ${ }^{\mathrm{b}, *}$, Jianping Wang ${ }^{\mathrm{a}}$, Hugh Gong ${ }^{\mathrm{c}}$ \\ ${ }^{a}$ Fashion Institute, Donghua University, Shanghai 200051, China \\ ${ }^{b}$ College of Mechanical Engineering, Donghua University, Shanghai 201620, China \\ ${ }^{c}$ School of Materials, The University of Manchester, Manchester, M13 9PL, UK
}

\begin{abstract}
This study is devoted to recognizing the breathing resistances of wearing respirators from respiratory and surface electromyography (sEMG) signals. Ten subjects were required to sit for 5 min and walk for 5 min while wearing two different models of N95 filtering facepiece respirators (FFRs) and without a respirator. We recorded the sEMG signals from the respiratory muscles of the subjects, and the respiratory amplitude is also collected. Subsequently, fifteen features of the sEMG time domain and respiratory amplitude were extracted and used as input vectors to a recognition model based on artificial neural networks (ANNs). Finally, the experimental results show that these artificial neural networks are effective for recognizing different airway resistances of wearing respirators from sEMG and respiratory signals. The results also indicate that abdominal and scalene are the primary respiratory muscles affected by using N95 FFRs.

Relevance to industry: Respirator manufactures and administrations can readily employ this paper's findings for recognizing the breathing resistances of wearing respirators from respiratory and surface electromyography (sEMG) signals based on artificial neural networks automatically. Observations of the present study are in support of testing only the two primary muscles (abdominal and scalene) to simplify the evaluation of the effects of the breathing resistances of wearing respirators on respiratory muscles.
\end{abstract}

\footnotetext{
${ }^{*}$ Corresponding author

Email address: yzl@dhu.edu.cn (Zhongliang Yang)
} 
Keywords: Breathing resistance, Surface electromyography, N95 filtering facepiece respirators, Respiratory amplitude, Artificial neural networks

\section{Introduction}

Concerns over air pollution have highlighted the importance of respiratory protection for workers and the general public. As a common form of personal protective equipment (PPE), N95 filtering facepiece respirators (N95 FFR) are widely used for medical staff and workers in atmospherically hostile environment $[1,2]$. As is known to all, one of the primary reasons for workers disliking wearing respirators is discomfort [3]. In the last decades, greater attention has been paid to the physiological impact of the respirators on wearers $[4,5,6,7,8]$. Many researchers have been working on the following physiological indices: the cardiopulmonary effects of wearing the respirator $[9,7]$, resistance to breathing while wearing a respirator $[5,1]$, pressure [10], and heat stress [11] imposed by the use of a respirator. Breathing resistance with respirators has been identified as the cause of respiratory fatigue [12], which can be reflected by Surface electromyography (sEMG) signals of respiratory muscles and respiratory signals [13, 14]. However, there is little research concerning respiratory muscle responses to the use of a respirator.

We focus on the respiratory muscle and respiratory signal responses to the different airway resistances [7], which can be changed with different work intensities and filter resistances of respirators. According to former research [5], as work intensity increased, an increase in breathing resistance was found. Lee and Wang [1] also indicated that the use of N95 respirator increased the inspiratory and expiratory flow resistances. In our previous work [13], the results of the statistical analysis showed that the physiological responses to breathing resistance of wearing an N95 FFR for 5 min in sitting and walking are relatively small. However, the relationship between respiratory muscle and respiratory signal responses and breathing resistances are very difficult to describe quantitatively by traditional mathematics or mechanic due to the nonlinearity of the parameters and a large number of variables involved. This, however, seems to be an ideal situation for the application of artificial neural networks (ANNs), which are developed to tackle problems with large numbers of nonlinear variables [15].

An ANN imitates the behavior of biological neural networks to develop solutions to problems from the data provided to it. Neural networks take 
previously solved examples and look for patterns, learn these patterns, and develop the ability to correctly classify new patterns (i.e. provide forecasts/predictions) [16]. The neural network learns by adjusting the interconnection weights between layers. ANNs have shown accurate performance in different classification tasks. Attempts have recently been made to apply the ANN technique to clothing ergonomics and comfort. For example, Luo et al. [17] constructed a fuzzy neural network model for predicting clothing thermal comfort. Wong et al. [18] used ANN for Predictions of Human Psychological Perceptions of Clothing Sensory Comfort. At the same time, ANNs has been of considerable interest for classifying sEMG signals [19, 20, 21, 22]. The applications of ANNs in these areas show great promise because an ANN can deal with the nonlinearity of problems, detect patterns and relationships in the data, and interpret information from a lot of variables [15].

In this paper, we investigate the use of the ANN to recognize the breathing resistance of respirators from sEMG and respiratory signals. The purpose of this work is to verify the possibility of using non-linear techniques in this area and find out the main respiratory muscle affected during the use of N95 FFRs. The hypothesis is that ANN can be used to recognize the breathing resistance of respirators from sEMG and respiratory signals. A series of objective experiments were conducted to assess physiological changes, that is, sEMG signals and respiratory signals, with the use of N95 FFRs on actual human subjects in two conditions, which were sitting and walking.

\section{Materials and methods}

\subsection{Participants}

This study was approved by the Ethics Committee of Donghua University. Ten healthy men volunteered to participate in the experiment $(M=26$, $\mathrm{SD}=1.8$, range from 25 to 32 years), the majority of whom $(8 / 10)$ were experienced N95 FFR users. All participants had a medical examination to eliminate any subject with respiratory muscle diseases and respiratory infection. After introducing the nature, purpose, methods and the risk of the study to the participants, they were required to complete a background questionnaire about personal information such as height $(\mathrm{M}=173.5, \mathrm{SD}=2.8$, range from 168 to $178 \mathrm{~cm})$ and weight $(\mathrm{M}=69.5, \mathrm{SD}=2.7$, range from 65 to $72 \mathrm{~kg})$. 


\subsection{N95 FFRs selection}

In this study, we selected two models of N95 FFRs. FFRA (3M 8210, N95 FFR) and FFRB (3M 8210v, N95 FFR/EV) were used, and the EV opens to release exhaled air and closes during inhalation. They are designed to fit all users [23]. The presence of an EV is intended to affect breathing resistance by reducing exhalation pressure. Adding a valve typically lowers the exhalation pressure drop by 50\%. Thus, two models of N95 FFR are all cup-shaped respirators and have different filter resistances. The selected respirators are shown in Figure 1.

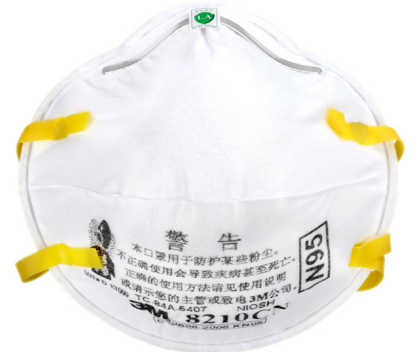

(a) $3 \mathrm{M} 8210$

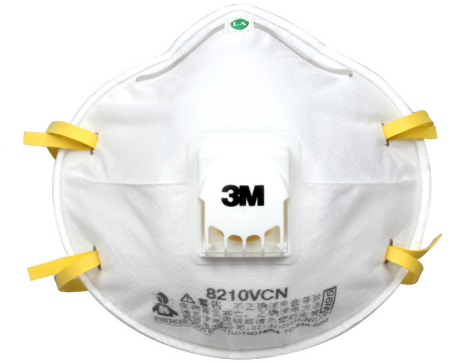

(b) $3 \mathrm{M} 8210 \mathrm{v}$

Figure 1: Tested respirators

\subsection{Tested respiratory muscles}

In the experiment, there is the issue of the proper choice of muscles and the corresponding location of the electrodes [24]. According to previous researches, the respiratory muscles include the diaphragm, the intercostal, the abdominal, the sternomastoid and scalene [13, 25, 26, 27].

\subsection{Experimental protocol}

The experiment contains six trials, and each trial differs in breathing resistance via changing work intensities and types of respirators $[5,1]$. The trials are shown in Table 1. The subject performed a walking on a treadmill with a speed of $1.6 \mathrm{~m} / \mathrm{s}$ [28], which is comfortable for an adult. All participants were required to sign a consent form with a detailed description of the experiment.

One week before this experiment, a fit test was performed to ensure that each subject had his personal respirator with an adequate size and was well 
Table 1: Types of trials

\begin{tabular}{lll}
\hline Types of trials & Respirator conditions & Motion conditions \\
\hline Trial 1 & No respirator & Sitting \\
Trial 2 & FFRA $(3 \mathrm{M} 8210$, N95 FFR) & Sitting \\
Trial 3 & FFRB (3M 8210v, N95 FFR/EV) & Sitting \\
Trial 4 & No respirator & Walking \\
Trial 5 & FFRA $(3 \mathrm{M} \mathrm{8210,} \mathrm{N95} \mathrm{FFR)}$ & Walking \\
Trial 6 & FFRB $(3 \mathrm{M} \mathrm{8210v,} \mathrm{N95} \mathrm{FFR/EV)}$ & Walking \\
\hline
\end{tabular}

fitted without leaking air. For the NIOSH bivariate panel, small headforms were defined as those falling in cells 1-3, medium headforms were those falling in cells 4-7, and large headforms were those falling in cells 8-10 [29]. The experimental results of Lei et al. [30] also showed that the combinations of 4-6 cells (medium headforms) and 3M 8210 respirator were matching. Thus, the subjects with medium headforms were selected in this experiment.

The duration of each trial is 5 minutes. During experimental phase, a subject entered the climate chamber controlled at an air temperature of 25 and a relative humidity of $70 \%$. After cleaning the skin with alcohol and applying electrolyte gel, the subject was fitted with respiratory and sEMG sensors. The placement of sensors can be seen in the Figure 2. Before wearing a respirator, the subject took a rest for $30 \mathrm{~min}$ on a chair. After being acclimatized to the respirator for a minimum of a 5-min period, he was required to breathe for 5 -min during each trial. Between two trials, the respirator was removed and the subject was required to take a rest for 20 min. The six 5-min trials were performed randomly. After completing the six trials, the respirator was removed.

\subsection{Data acquisition}

We used a 14-channel digital system (ZJE-II, ZJE Studio Ltd., China) for collecting, amplifying and transmitting respiratory and sEMG signals. The respiration sensor (ZJE Studio Ltd., China), which includes an easy fitting high durability latex rubber band fixed with a self-adhering belt, monitors the respiratory amplitude. The sEMG sensors (ZJE Studio Ltd., China) can detect sEMG signals from 0 to $2000 \mu \mathrm{V}$. The raw signals were sampled at 1000 samples/s and band-pass filtered at 10-500 $\mathrm{Hz}$ with a notch filter implemented to remove the $50 \mathrm{~Hz}$ line interference. 


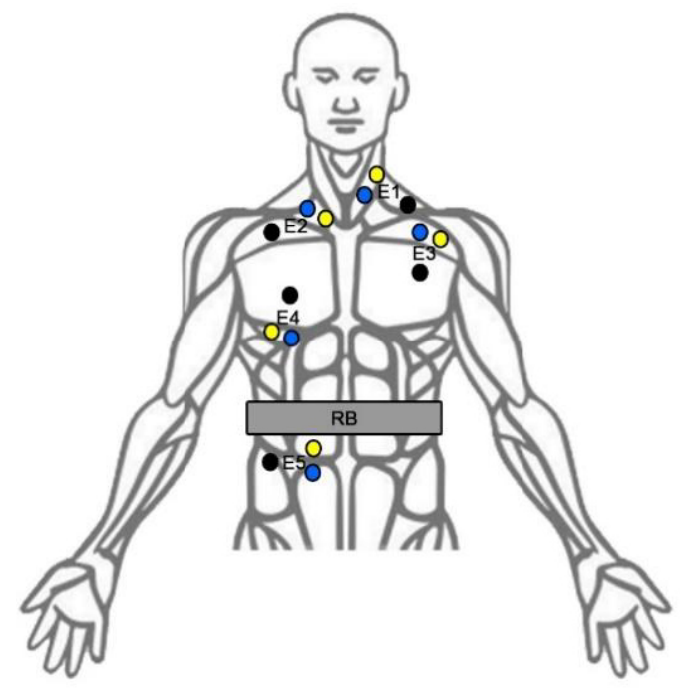

Figure 2: The placement of sensors. E1, sternomastoid electrodes; E2, scalene electrodes; E3, intercostal electrodes; E4, diaphragm electrodes; E5, abdominal electrodes; RB, respiration band, around the abdomen. Here, yellow represents negative electrode, blue represents positive electrode, black represents common electrode.

The disposable electrodes (Jun Kang Medical Supplies Ltd., China) made of silver/silver chloride electrodes (10 $\mathrm{mm}$ in diameter), conductive paste and backing (non-woven fabric, foam) composition ( $50 \mathrm{~mm}$ in diameter) were used to measure sEMG activity. Before electrode attachment, alcohol was used to clean the skin, and the conductive gel was used to improve the contact of the electrode with the skin [31]. A photograph of data acquisition session can be illustrated in Figure 3.

\subsection{Feature extraction}

From respiratory signals, respiratory amplitude $(\mathrm{RA}, \mu \mathrm{V})$ was recorded as an input parameter. The RA is measured by calculating the difference between the highest and lowest points in one breath [32]. We can collect one parameter per participant per breath from respiratory signals.

From sEMG signals, time domain features such as average EMG amplitude (aEMG), variance (VAR) and Root Mean Square (RMS) [33, 34, 35] were recorded as input parameters. The formulas are as follows:

$$
a E M G=\frac{\sum_{i=1}^{N}\left(X_{i}\right)}{N}
$$




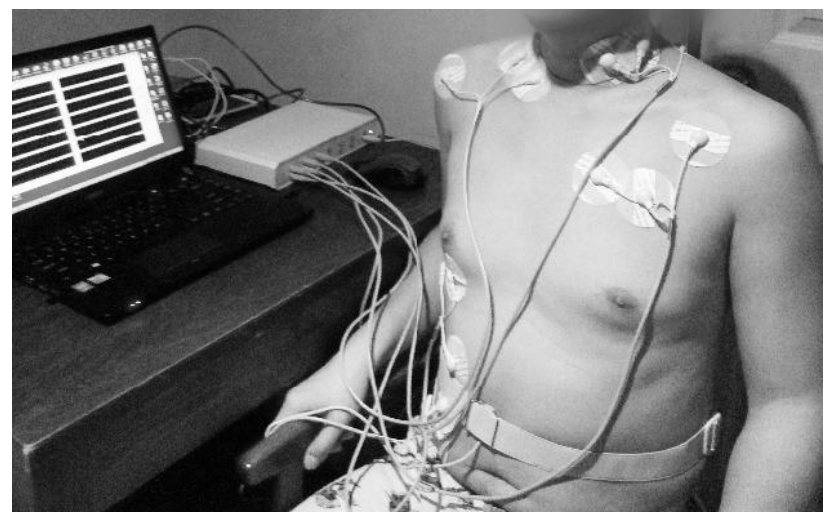

Figure 3: A photograph of data acquisition session

$$
\begin{gathered}
V A R=\frac{\sum_{i=1}^{N}\left(X_{i}-a E M G\right)^{2}}{N} \\
R M S=\sqrt{\frac{\sum_{i=1}^{N}\left(X_{i}\right)^{2}}{N}}
\end{gathered}
$$

where $X_{i}$ is the $i$ th sampling value of EMG signals in one sampling period.

These features of sEMG signals were extracted during each epoch. The beginning of the inspiration in one breath was set as the epoch onset, and the ending of the expiration of the breath was set as the epoch endpoint. We can collect a number of time domain characteristics $(3) \times$ Number of tested muscles (5), amounting to 15 parameters per participant per breath from sEMG signals. Thus, we can collect totally 16 parameters per breath per participant from respiratory and sEMG signals.

\subsection{Artificial neural network construction}

We used the feed-forward multilayer perceptron (MLP) neural networks for this work. MLP neural networks are the most commonly used feedforward neural networks due to their fast operation, ease of implementation, and smaller training set requirements [36, 37]. MLPs are normally trained with the back-propagation algorithm. The back-propagation rule propagates the errors through the network and allows adaptation of the hidden parameters. The number of input and output vectors depends on how many parameters or variables are provided to and expected from the ANN. The weights in the neural networks are used to transmit activity between inputs and outputs by means of transfer functions within the network. They are modified 
during the learning cycles, and the significance of each input is reinforced or weakened by adjusting the weight according to its activity [15].

As one of the most common artificial neural networks (ANNs), MLP has been widely used in pattern recognition models for sEMG signals [38]. A three-layer network consisting of one input layer, one hidden layer with a sigmoid function, and one output layer with a hyperbolic tangent (TanH) function was used to set up the MLP classifier.

Two critical characteristics of the MLP are: the nonlinear processing elements (PEs) which have a nonlinearity that must be smooth (the sigmoid function and the TanH function are the most widely used); and their massive interconnectivity (i.e. any element of a given layer feeds all the elements of the next layer) [39]. In our study, the activation function which is the sigmoid function for the hidden layer is given by:

$$
f(x)=\frac{1}{1+e^{-a x+b}}
$$

The activation function which is the TanH function for the output layer is given by:

$$
g(x)=\frac{e^{x}-e^{-x}}{e^{x}+e^{-x}}
$$

The MLP is trained with error correction learning, which works in the following way: From the model response at PE $j$ at iteration $k, y_{j}(k)$, and the desired response $d_{j}(k)$ for a given input pattern an instantaneous error $e_{j}(k)$ is defined by

$$
e_{j}(k)=d_{j}(k)-y_{j}(k)
$$

Using the gradient descent learning, each weight in the ANN can be adapted by correcting the present value of the weight with a term that is proportional to the present input and error at the weight, i.e.

$$
w_{j l}(k+1)=w_{j l}(k)+\eta \delta_{j}(k) x_{l}(k)
$$

where the local error $\delta_{j}(k)$ can be directly computed from $e_{j}(k)$ at the output $\mathrm{PE}$ or can be computed as a weighted sum of errors at the internal PEs. The constant $\eta$ is the step size. The procedure represents backpropagation (BP) algorithm. Momentum learning is used to speed up and stabilize convergence, and the equation to update the weights becomes

$$
w_{j l}(k+1)=w_{j l}(k)+\eta \delta_{j}(k) x_{l}(k)+\alpha\left(w_{j l}(k)-w_{j l}(k-1)\right)
$$


where $\alpha$ is the momentum.

To start the BP, a small random value is set as an initial value for each weight, and the $\mathrm{BP}$ proceeds until the termination criterion is met. In our study, two termination criteria were set for the training phase: the maximum number of iterations was 2000, and the minimum mean square error (MSE) was less than 0.01. If either criterion were satisfied, the training would stop.

To investigate the effects of the different respiratory muscles on the recognition performance of ANN, we constructed the MLP recognition models for each respiratory muscle. Seven sets of parameters were used as input vectors to construct seven MLP-based recognition models respectively, as shown in Table 2. Taking the input set of all 16 parameters as the example, the basic structure of the proposed MLP-based recognition model is shown in Figure 4. The training and testing of the MLP model were constructed in the simulation software NeuroSolutions 6 on Windows 7 .

Table 2: The Parameters of the seven MLP-based recognition models

\begin{tabular}{lll}
\hline Recognition models & Input sets & Input vectors \\
\hline 1 & Respiratory parameter & RA \\
2 & Abdominal parameters & RMS, VAR and aEMG of abdominal \\
3 & Scalene parameters & RMS, VAR and aEMG of scalene \\
4 & Diaphragm parameters & RMS, VAR and aEMG of diaphragm \\
5 & Intercostal parameters & RMS, VAR and aEMG of intercostal \\
6 & Sternomastoid parameters & RMS, VAR and aEMG of sternomastoid \\
7 & All 16 parameters & RMS, VAR and aEMG of 5 muscles, RA \\
\hline
\end{tabular}

In this paper, accuracy rate $(\mathrm{AR})$ is adopted to evaluate the recognition effect of the model. The algorithm can be written as follows:

$$
A R=\frac{m}{M} \times 100 \%
$$

where $m$ is the number of samples recognized correctly, and $M$ is the total number of samples.

\section{Results and discussions}

\subsection{Results of feature extraction}

Because of different respiratory rates of each trial per participant, there were different epochs/breaths for six trials and ten participants. The epochs 


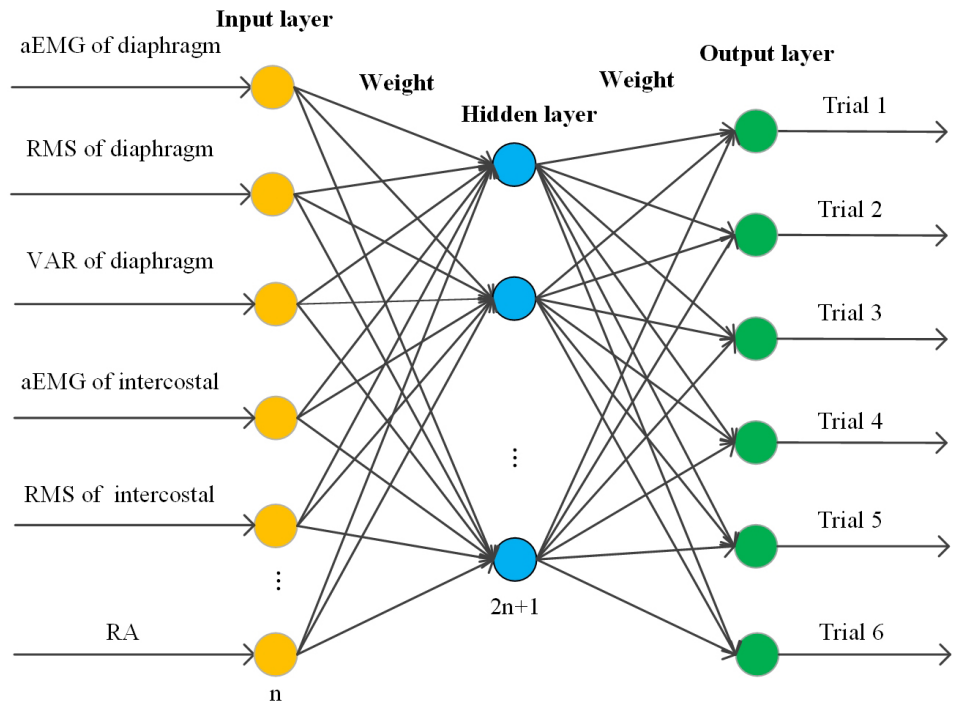

Figure 4: MLP-based breathing resistance recognition model

during 5 minutes for each subject are shown in Table 3. 100 epochs were randomly selected from each subject and each trial, amounting to 1000 epochs for each trial. 16 parameters were successfully extracted during each epoch. The dataset was randomly divided into two subsets, a training set and test set. We randomly selected $70 \%$ of the data as the training set and $30 \%$ as the test set.

Randomly selected respiratory signals and electromyograms for the six trials with different breathing resistances of wearing respirators are shown in Figure 5.

\subsection{Statistic analysis of the sEMG indices}

The averages of aEMG, VAR and RMS for the diaphragm, the intercostal, the abdominal, the sternomastoid and scalene muscles for the different trials are shown in Figure 6. These averages are the means of these indices for the ten participants. The error bars represent the standard error (SE). According to Figure 6, the error is small, meaning that the consistency of the data collected across participants is high. Thus, the recognition algorithms can be performed on the data across subjects. 
Table 3: Number of epochs during 5 minutes for 10 subjects

\begin{tabular}{lllllll}
\hline \multirow{2}{*}{ Subjects } & \multicolumn{7}{l}{ Number of epochs during 5 minutes } \\
\cline { 2 - 7 } & Trial & Trial 2 & Trial 3 & Trial 4 & Trial 5 & Trial 6 \\
\hline 1 & 120 & 110 & 115 & 235 & 220 & 230 \\
2 & 119 & 111 & 116 & 236 & 221 & 234 \\
3 & 121 & 112 & 117 & 237 & 221 & 231 \\
4 & 120 & 108 & 115 & 235 & 222 & 232 \\
5 & 122 & 107 & 114 & 230 & 223 & 233 \\
6 & 118 & 109 & 118 & 234 & 219 & 229 \\
7 & 119 & 110 & 113 & 233 & 218 & 227 \\
8 & 120 & 112 & 116 & 238 & 220 & 228 \\
9 & 122 & 113 & 117 & 235 & 221 & 231 \\
10 & 121 & 108 & 115 & 234 & 217 & 230 \\
\hline
\end{tabular}

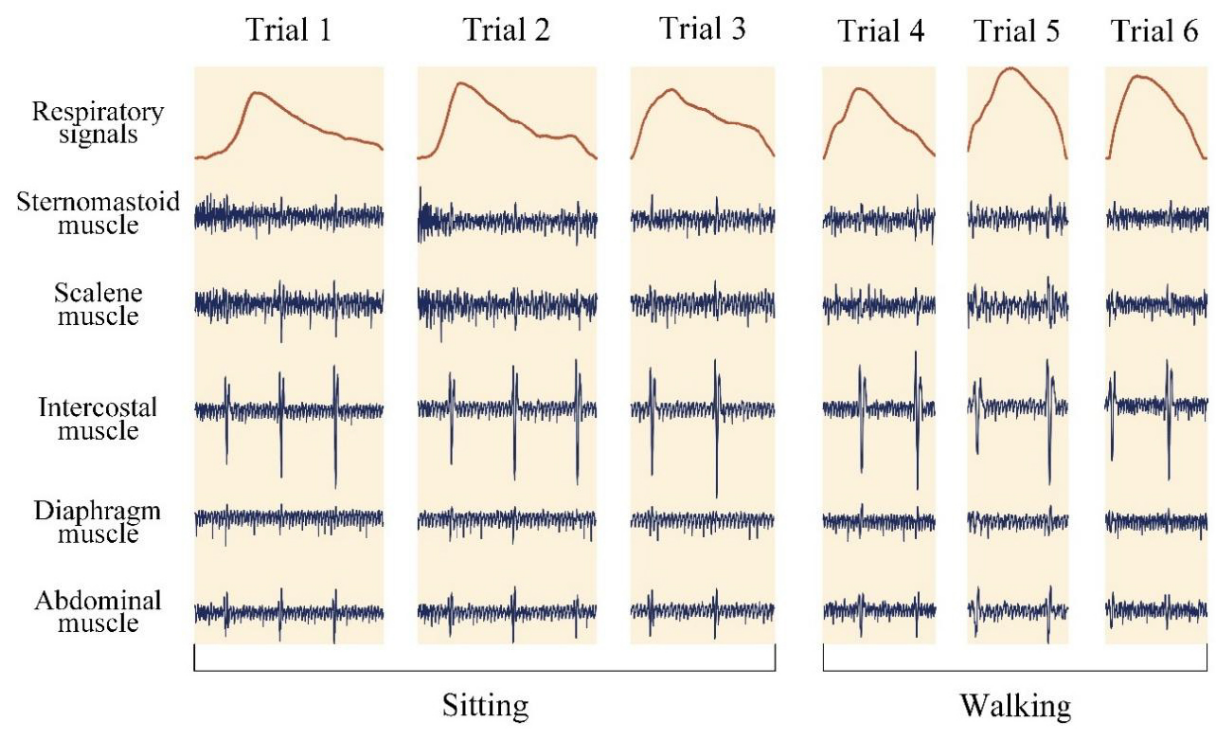

Figure 5: Respiratory signals and electromyograms of six trials across participants, each panel illustrating a single breath taken from a single participant

\subsection{Classification results}

The confusion matrixes of the classifications using different sets of input vectors based on the MLP classifier are shown in Figure 7. 

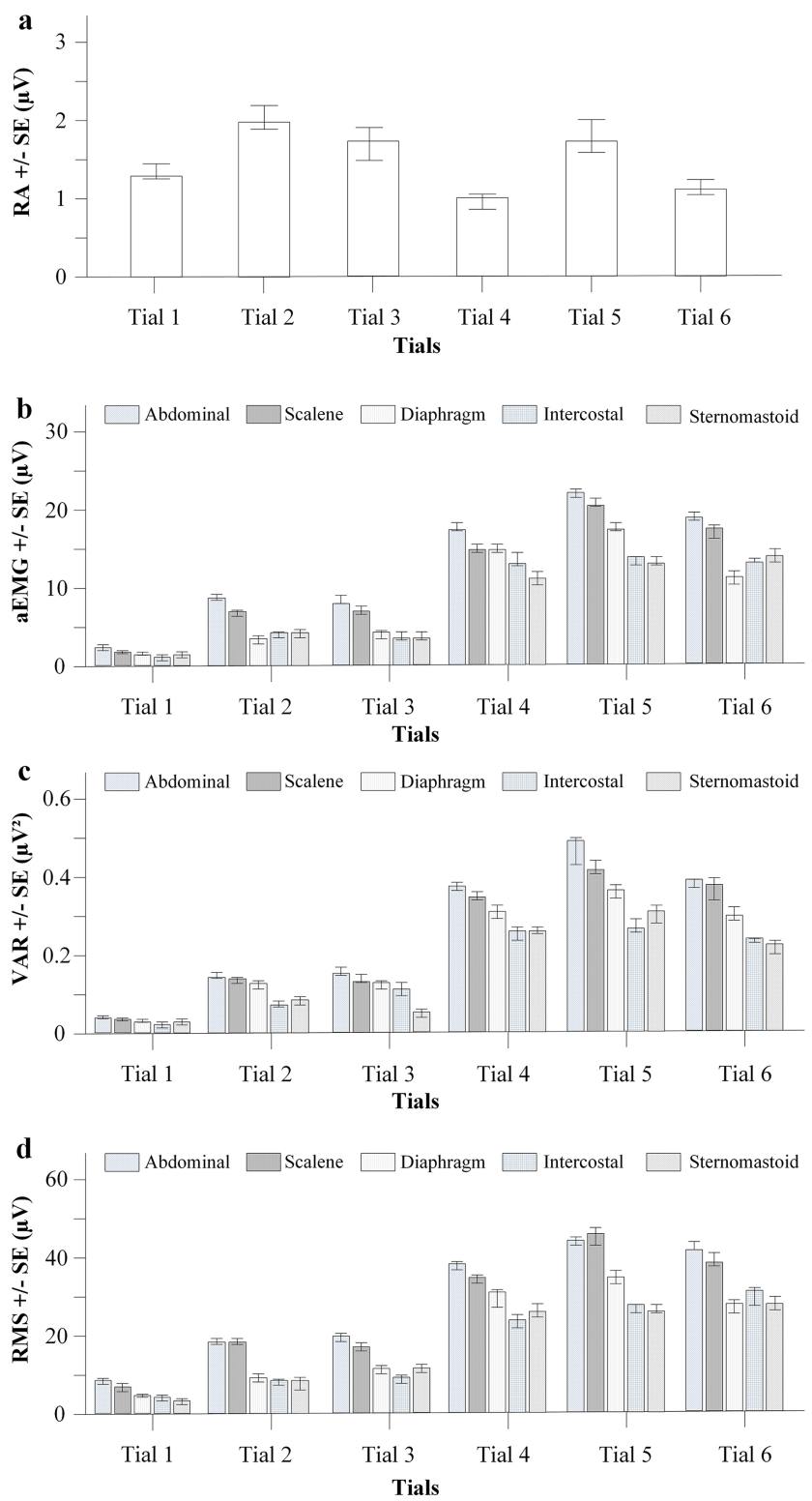

Figure 6: Histogram bars and error bars of 16 parameters (Error bar denote SE of the means). (a) Histogram bars and error bars of RA, (b) histogram bars and error bars of aEMG, (c) histogram bars and error bars of VAR, and (d) histogram bars and error bars of RMS 


\subsection{Statistic analysis of accuracy rates (ARs)}

To check for statistical significance, one-way ANOVA was performed on classification accuracy rates (ARs) of seven MLP-based recognition models. The significance level represented by $\alpha$ is selected as 0.05 . The result of this test shows statistically significant differences among these recognition models $(p=0.000)$. The results of post-hoc Tukey-Kramer tests were shown in Figure 8.

Table 4: The Parameters of the six MLP-based recognition models

\begin{tabular}{lll}
\hline Recognition models & aAR for the training set $(\%)$ & aAR for the test set $(\%)$ \\
\hline Respiratory amplitude & 71.75 & 70.82 \\
abdominal & 62.92 & 62.28 \\
Scalene & 61.57 & 60.83 \\
Diaphragm & 58.3 & 57.55 \\
Intercostal & 54.32 & 53.12 \\
Sternomastoid & 50.18 & 48.87 \\
All 16 parameters & 81.52 & 80.55 \\
\hline
\end{tabular}

aAR, average accuracy rate.

The average recognition accuracy for seven MLP-based recognition models is shown in Table 4. As shown in Figure 8 and Table 4, recognition model with all 16 parameters significantly has the highest recognition accuracy, followed by recognition model with respiratory amplitude, recognition model with abdominal parameters, recognition model with scalene parameters, recognition model with diaphragm parameters, recognition model with intercostal parameters, and recognition model with sternomastoid parameters. Further, for the five respiratory muscles, abdominal was not significantly better than scalene, scalene was not significantly better than the diaphragm, the diaphragm was not significantly better than intercostal, and intercostal was not significantly better than sternomastoid.

\subsection{Discussion}

Surface EMG-based approaches have been successfully used for assessing muscle activity [27] and fatigue [40] with linear [41] and non-linear [42] techniques. These researchers developed clinically viable activity and fatigue assessment strategies. However, to our knowledge, there are limited studies 
published to assess respiratory muscle activity and fatigue caused by wearing respirators. In our previous work [13], we firstly used statistics to assess the physiological responses (respiratory signals and sEMG signals) to different breathing resistances of wearing respirators. As a continuation of our previous work, this paper is the first reported study that presents a systematic comparison of different input parameters for recognition of six trials with different breathing resistances of wearing respirators from respiratory and sEMG signals using artificial neural networks.

The results suggest that our MLP-based model can be used for recognizing six trials of different breathing resistances from physiological signals. The results supported the hypothesis that ANN can be used to recognize the breathing resistance of respirators from sEMG and respiratory signals. The basis of this achievement is revealed by the graphs of respiratory signals and electromyograms Figure 5 which show that the respiratory signals and respiratory muscles have different amplitudes when participants bearing different breathing resistances.

Using all 16 parameters as input vectors, the MLP model was able to have the highest recognition accuracy. The finding is in accordance with Linderman et al. [43], who pointed out that the recognition accuracy was improved by increasing the muscle numbers. Interestingly, recognition model using respiratory amplitude has a relatively higher recognition accuracy than remaining 5 recognition models with different sEMG parameters of respiratory muscles (Figure 7 and Figure 8, Table 4). This finding is also in alignment to our previous research [13], which claimed that the respiratory amplitude has the highest Pearsons correlation coefficient with subjective assessment of overall breathing resistance. Moreover, among the five muscles, the recognition models of abdominal and scalene parameters had the highest recognition accuracy (Figure 7 and Figure 8, Table 4). The finding is also in accordance with our previous study [13], which also indicated that they are the primary muscle affected during the use of N95 FFRs. However, the findings that we got from statistical analysis of previous research [13] is more ambiguous than those we get from this study that used artificial neural networks. Thus, it can be concluded that ANN is more effective for measuring the relationship between physiological signals and breathing resistance changes of wearing respirators than statistics (MANOVA) [13].

Nevertheless, our findings and the general approach have several limitations and challenges:

(1) In this study, we used the most common ANN, that is, MLP, for 


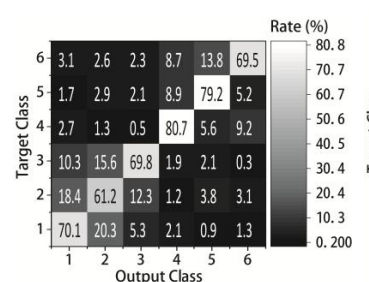

(a)

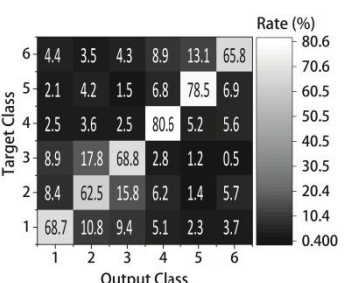

(b)

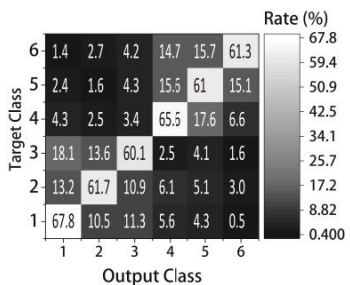

(c)

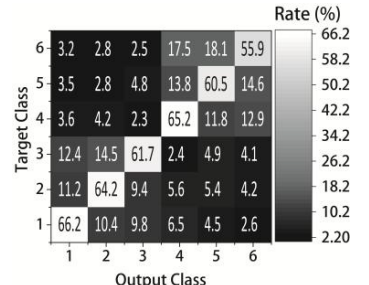

(d)

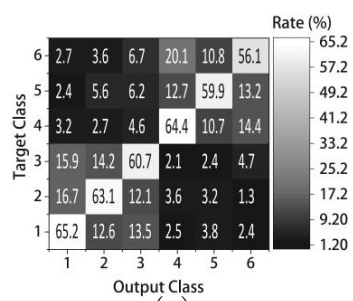

(e)

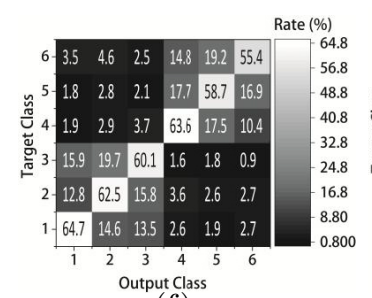

(f)

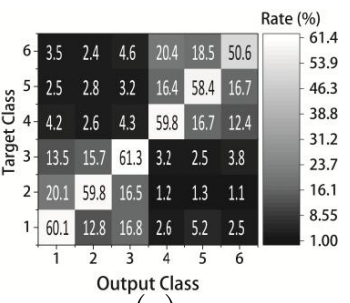

(g)

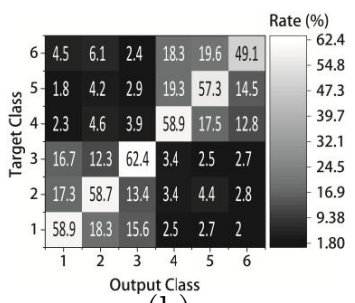

(h)

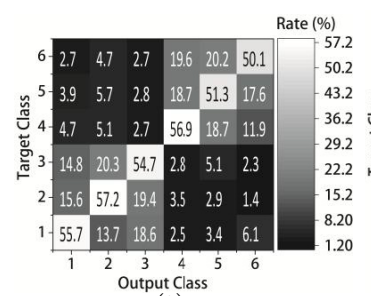

(i)

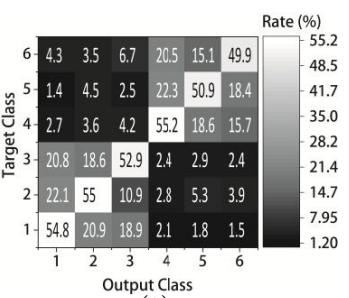

(j)

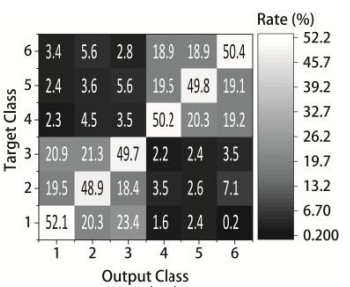

(k)

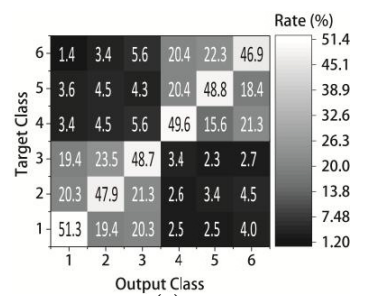

(1)

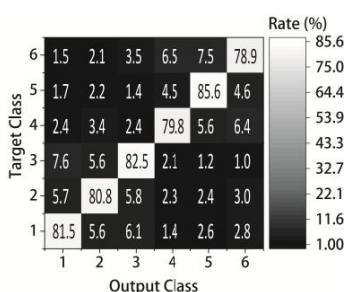

(m)

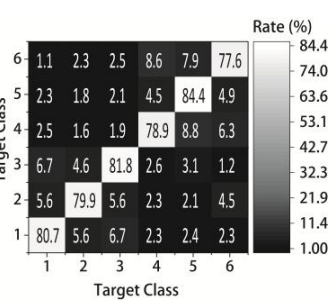

(n)

Figure 7: Confusion matrixes of the classifications using seven sets of parameters as input vectors $(1$, Trial $1 ; 2$, trial $2 ; 3$, trial $3 ; 4$, trial $4 ; 5$, trial $5 ; 6$, trial 6 ). (a) Respiratory amplitude for the training set, (b) respiratory amplitude for the test set, (c) abdominal parameters for the training set, (d) abdominal parameters for the test set, (e) scalene parameters for the training set, (f) scalene parameters for the test set, (g) diaphragm parameters for the training set, (h) diaphragm parameters for the test set, (i) intercostal parameters for the training set, $(\mathrm{j})$ intercostal parameters for the test set, $(\mathrm{k})$ sternomastoid parameters for the training set, (l) sternomastoid parameters for the test set, (m) all 16 parameters for the training set, and (n) all 16 parameters for the test set. 


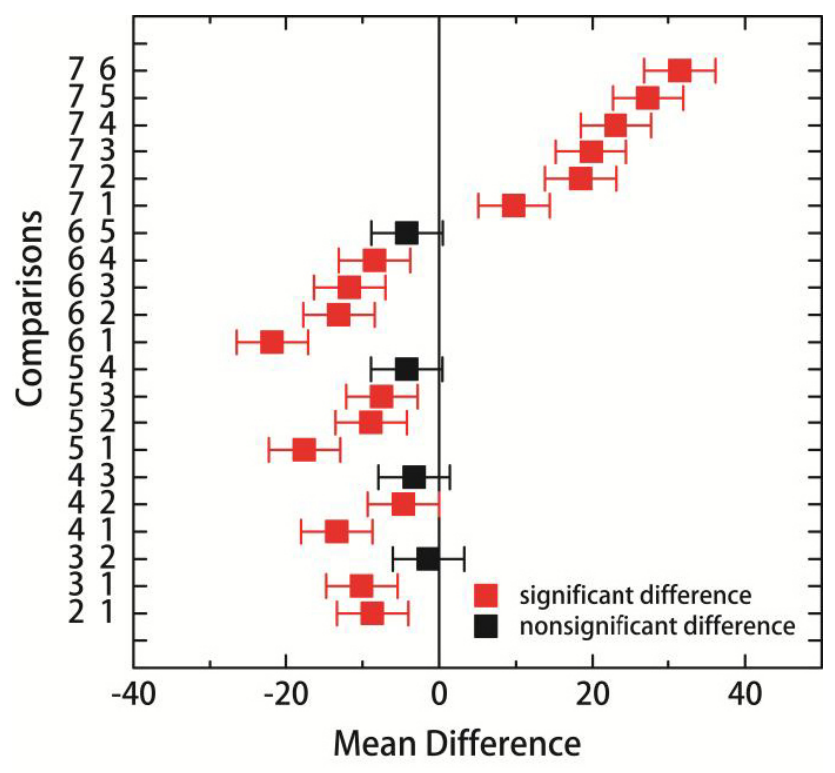

Figure 8: Means Comparison Plot of post-hoc Tukey-Kramer tests. 1, recognition model with respiratory amplitude; 2 , recognition model with abdominal parameters; 3 , recognition model with scalene parameters; 4 , recognition model with diaphragm parameters; 5 , recognition model with intercostal parameters; 6 , recognition model with sternomastoid parameters; 7 , recognition model with all 16 parameters.

recognition model construction. There are many other kinds of ANN can be used for further research, such as Elman neural networks [44, 45], Radial Basis Function networks [38] and Learning Vector Quantization networks [46]. In future studies, we plan to use other advanced classifiers to see whether the performance of our recognition method can be further improved.

(2) We only used two models of N95 respirators (3M 8210 and 3M 8210v), so more other models having obviously different $\mathrm{mmHg}$ pressure drops can be used for future research. As an approximate values, 3M 8210 is around 8-9 $\mathrm{mmH}_{2} \mathrm{O}$ at $85 \mathrm{~L} / \mathrm{min}$ of constant airflow, and $3 \mathrm{M} 8510$ is around 5-6 $m m \mathrm{H}_{2} \mathrm{O}$ at $85 \mathrm{~L} / \mathrm{min}$ of constant airflow.

(3) We only tested two kinds of work intensity (sitting still and walking). In future work, we should test on more kinds of work intensity.

(4) Only a few subjects were tested in our experiment. In future research, we plan to collect sEMG signals from participants covering a range of ages, countries, races, body types and of both genders, to increase the 
generalizability of the findings.

(5) We only used sEMG amplitude-based parameters as input vectors. To further improve the recognition accuracy, sEMG parameters based on spectral analysis, sEMG parameters based on timefrequency distributions and non-linear parameters could be additionally used.

\section{Conclusions}

Our work has demonstrated that it is possible to recognize breathing resistances of wearing respirators by using artificial neural networks. This study also indicated that respiratory amplitude, abdominal and scalene muscles are more sensitive to the changes of breathing resistances of wearing respirators. While the results are encouraging, additional research is needed to further develop the method. Our future work will concentrate on the evaluation of longer periods of FFR wear. In addition, subjective ratings by the test subjects will also be obtained and compared with the resistance and sEMG data.

\section{Conflict of Interest Statement}

The authors declare that the research was conducted in the absence of any commercial or financial relationships that could be construed as a potential conflict of interest.

\section{Funding}

The authors would like to thank the participants of the experiment. This study was partly supported by the National Natural Science Foundation of China (No. 51305077), the Fundamental Research Funds for the Central Universities (NO. CUSF-DH-D-2016068), the Zhejiang Provincial Key Laboratory of integration of healthy smart kitchen system (2014E10014), and the China Scholarship Council (CSC). Grant Numbers: 201506630036, 201506635030.

\section{References}

[1] H. P. Lee, D. Y. Wang, Objective assessment of increase in breathing resistance of n95 respirators on human subjects, Annals of occupational hygiene 55 (8) (2011) 917-921. 
[2] Y. Chen, J. Wang, Z. Yang, The human factors/ergonomics studies for respirators: a review and future work, International Journal of Clothing Science and Technology 27 (5) (2015) 652-676.

[3] A. M. J. A. Gutierrez, M. D. Galang, R. R. Seva, M. C. Lu, D. R. S. Ty, Designing an improved respirator for automotive painters, International Journal of Industrial Ergonomics 44 (1) (2014) 131-139.

[4] Y. P. Guo, L. Yi, H. Tokura, T. K. S. Wong, J. W. Y. Chung, M. D. I. Gohel, P. H.-m. Leung, E. Newton, Evaluation on masks with exhaust valves and with exhaust holes fromphysiological and subjective responses, Journal of physiological anthropology 27 (2) (2008) 93-102.

[5] J. G. Jones, The physiological cost of wearing a disposable respirator, The American Industrial Hygiene Association Journal 52 (6) (1991) 219 225 .

[6] R. J. Roberge, A. Coca, W. J. Williams, J. B. Powell, A. J. Palmiero, Physiological impact of the $n 95$ filtering facepiece respirator on healthcare workers, Respiratory Care 55 (5) (2010) 569-577.

[7] R. J. Roberge, J.-H. Kim, J. B. Powell, R. E. Shaffer, C. M. Ylitalo, J. M. Sebastian, Impact of low filter resistances on subjective and physiological responses to filtering facepiece respirators, PloS one 8 (12) (2013) e84901.

[8] B. E. Shykoff, D. E. Warkander, Physiologically acceptable resistance of an air purifying respirator, Ergonomics 54 (12) (2011) 1186-1196.

[9] J.-H. Kim, S. M. Benson, R. J. Roberge, Pulmonary and heart rate responses to wearing $n 95$ filtering facepiece respirators, American journal of infection control 41 (1) (2013) 24-27.

[10] S. Snook, W. Hinds, W. A. Burgess, Respirator comfort: Subjective response to force applied to the face, American Industrial Hygiene Association Journal 27 (2) (1966) 93-97.

[11] I. S. Laird, R. J. Pack, D. H. Carr, A survey on the use and non-use of respiratory protective equipment in workplaces in a provincial new zealand city, Annals of Occupational Hygiene 37 (4) (1993) 367-376. 
[12] E. J. Sinkule, J. B. Powell, F. L. Goss, Evaluation of n95 respirator use with a surgical mask cover: effects on breathing resistance and inhaled carbon dioxide, Annals of occupational hygiene 57 (3) (2013) 384-398.

[13] Y. Chen, Z. Yang, J. Wang, H. Gong, Physiological and subjective responses to breathing resistance of $n 95$ filtering facepiece respirators in still-sitting and walking, International Journal of Industrial Ergonomics 53 (2016) 93-101.

[14] W. Jian, Some advances in the research of semg signal analysis and its application, Sports Science 20 (4) (2000) 56-60.

[15] R. Gong, Y. Chen, Predicting the performance of fabrics in garment manufacturing with artificial neural networks, Textile research journal 69 (7) (1999) 477-482.

[16] M. Kolich, N. Seal, S. Taboun, Automobile seat comfort prediction: statistical model vs. artificial neural network, Applied ergonomics 35 (3) (2004) 275-284.

[17] X. Luo, W. Hou, Y. Li, Z. Wang, A fuzzy neural network model for predicting clothing thermal comfort, Computers \& Mathematics with Applications 53 (12) (2007) 1840-1846.

[18] A. Wong, Y. Li, P. Yeung, P. Lee, Neural network predictions of human psychological perceptions of clothing sensory comfort, Textile Research Journal 73 (1) (2003) 31-37.

[19] E. El-Daydamony, M. El-Gayar, F. Abou-Chadi, A computerized system for semg signals analysis and classifieation, in: Radio Science Conference, 2008. NRSC 2008. National, IEEE, 2008, pp. 1-7.

[20] K. A. Farry, I. D. Walker, R. G. Baraniuk, Myoelectric teleoperation of a complex robotic hand, Robotics and Automation, IEEE Transactions on 12 (5) (1996) 775-788.

[21] T. Itou, M. Terao, J. Nagata, M. Yoshida, Mouse cursor control system using emg, in: Engineering in Medicine and Biology Society, 2001. Proceedings of the 23rd Annual International Conference of the IEEE, Vol. 2, IEEE, 2001, pp. 1368-1369. 
[22] G. R. Naik, D. K. Kumar, M. Palaniswami, Multi run ica and surface emg based signal processing system for recognising hand gestures, in: Computer and Information Technology, 2008. CIT 2008. 8th IEEE International Conference on, IEEE, 2008, pp. 700-705.

[23] Z. Lei, J. Yang, Methodology for simulating air leakages of an n95 filtering facepiece respirator-a pilot study, Computer-Aided Design and Applications 9 (1) (2012) 43-53.

[24] S. P. Arjunan, D. K. Kumar, Recognition of facial movements and hand gestures using surface electromyogram (semg) for hci based applications, in: Digital Image Computing Techniques and Applications, 9th Biennial Conference of the Australian Pattern Recognition Society on, IEEE, 2007, pp. 1-6.

[25] I. Cossette, P. Monaco, A. Aliverti, P. Macklem, Respiratory muscle recruitment and their correlates with pulmonary volumes and flute musical tasks, in: 10ème Congrès Français d'Acoustique, 2010.

[26] M. Green, J. Moxham, The respiratory muscles, Clinical Science 68 (1) (1985) 1-10.

[27] E. Maarsingh, L. Van Eykern, A. Sprikkelman, M. Hoekstra, W. Van Aalderen, Respiratory muscle activity measured with a noninvasive emg technique: technical aspects and reproducibility, Journal of Applied Physiology 88 (6) (2000) 1955-1961.

[28] R. W. Bohannon, Comfortable and maximum walking speed of adults aged 2079 years: reference values and determinants, Age and ageing 26 (1) (1997) 15-19.

[29] Z. Zhuang, D. Groce, H. W. Ahlers, W. Iskander, D. Landsittel, S. Guffey, S. Benson, D. Viscusi, R. E. Shaffer, Correlation between respirator fit and respirator fit test panel cells by respirator size, Journal of occupational and environmental hygiene 5 (10) (2008) 617-628.

[30] Z. Lei, J. Yang, Z. Zhuang, R. Roberge, Simulation and evaluation of respirator faceseal leaks using computational fluid dynamics and infrared imaging, Annals of occupational hygiene 57 (4) (2013) 493-506. 
[31] C. J. De Luca, Use of the surface emg signal for performance evaluation of back muscles, Muscle \& nerve 16 (2) (1993) 210-216.

[32] A. G. Money, H. Agius, Analysing user physiological responses for affective video summarisation, Displays 30 (2) (2009) 59-70.

[33] U. Baspinar, H. S. Varol, V. Y. Senyurek, Performance comparison of artificial neural network and gaussian mixture model in classifying hand motions by using semg signals, Biocybernetics and Biomedical Engineering 33 (1) (2013) 33-45.

[34] J. Potvin, Effects of muscle kinematics on surface emg amplitude and frequency during fatiguing dynamic contractions, Journal of Applied Physiology 82 (1) (1997) 144-151.

[35] H. Ren, G. Xu, S. Kee, Subject-independent natural action recognition, in: Automatic Face and Gesture Recognition, 2004. Proceedings. Sixth IEEE International Conference on, IEEE, 2004, pp. 523-528.

[36] Y. Kocyigit, A. Alkan, H. Erol, Classification of eeg recordings by using fast independent component analysis and artificial neural network, Journal of Medical Systems 32 (1) (2008) 17-20.

[37] E. D. Übeyli, Combined neural network model employing wavelet coefficients for eeg signals classification, Digital Signal Processing 19 (2) (2009) 297-308.

[38] G. Tsenov, A. Zeghbib, F. Palis, N. Shoylev, V. Mladenov, Neural networks for online classification of hand and finger movements using surface emg signals, in: Neural Network Applications in Electrical Engineering, 2006. NEUREL 2006. 8th Seminar on, IEEE, 2006, pp. 167171.

[39] I. NeuroDimension, Neurosolutions user's guide.

URL http://www.neurosolutions. com/neurosolutions/help/

[40] M. González-Izal, A. Malanda, E. Gorostiaga, M. Izquierdo, Electromyographic models to assess muscle fatigue, Journal of Electromyography and Kinesiology 22 (4) (2012) 501-512. 
[41] M. Gonzalez-Izal, I. Rodríguez-Carreño, A. Malanda, F. MallorGiménez, I. Navarro-Amézqueta, E. Gorostiaga, M. Izquierdo, semg wavelet-based indices predicts muscle power loss during dynamic contractions, Journal of Electromyography and Kinesiology 20 (6) (2010) 1097-1106.

[42] D. T. MacIsaac, P. A. Parker, K. B. Englehart, D. R. Rogers, Fatigue estimation with a multivariable myoelectric mapping function, Biomedical Engineering, IEEE Transactions on 53 (4) (2006) 694-700.

[43] M. Linderman, M. A. Lebedev, J. S. Erlichman, Recognition of handwriting from electromyography, PloS one 4 (8) (2009) e6791.

[44] M. Ardalani-Farsa, S. Zolfaghari, Chaotic time series prediction with residual analysis method using hybrid elman-narx neural networks, Neurocomputing 73 (13) (2010) 2540-2553.

[45] Y. Chen, Z. Yang, J. Wang, Eyebrow emotional expression recognition using surface emg signals, Neurocomputing 168 (2015) 871-879.

[46] T. Kohonen, Improved versions of learning vector quantization, in: Neural Networks, 1990., 1990 IJCNN International Joint Conference on, IEEE, 1990, pp. 545-550. 
LaTeX Source Files
Click here to download LaTeX Source Files: elsarticle.zip

LaTeX Source Files
Click here to download LaTeX Source Files: elsarticle.zip Click here to download LaTex Source Files: elsarticlezip

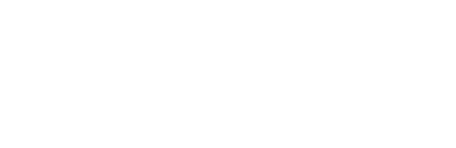
(1) (1)

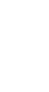
(1) (1) (1) (1) (1) (1) . . . . . . . . . . . . (1) 